sie genügende Grade erreichen und dadurch die Beziehungen zwischen Blut und Gewebe stark herabsetzen oder aufheben, anatomische Läsionen zur Folge haben; die diesen zugrunde liegenden Kreislaufstörungen treten zu Beginn der Erstwirkung auf Grund einer direkten Reizung, während der Erstund Zweitwirkung auf Grund einer von der Commotio hinterlassenen abnormen Erregbarkeit der Hirngefäßnerven, aus der eine Reizung hervorgeht, auf. Die nach Commotio sich einstellenden Funktionsstörungen der Hirntätigkeit haben somit keine anatomische (organische) Grundlage, sondern umgekehrt, die vorkommenden anatomischen Veränderungen des Hirnes beruhen anf funktionellen Vorgängen, nämlich der Gefäßnerven und Gefäße.

Diese Betrachtungsweise, in der das Nervensystem das erste Glied wie des physiologischen, so des pathologischen Geschehens ist, haben wir von je auf die anatomisehen Veränderungen überhaupt angewandt und ihre Notwendigkeit und Fruchtbarkeit sowohl anatomisch, besonders an experimentell gewonnenem Material, als rein experimentell, als theoretisch ${ }^{1}$ ) nachgewiesen.

\title{
XI.
}

\section{Über die Bildung von granulierten Leukozyten im Karzinomgewebe.}

\author{
(Aus der Hebammenschule in Straßburg i. E. Direktor: Prof. Dr. H. Freund.)
}

\author{
Von \\ Paul Weill. \\ (Hierzu Talel IV,)
}

Trotzdem über das Vorkommen von lymphoidem Gewebe und auch von leukozytären Zellen im Bindegewebe von Tumoren vieles veröffentlicht worden ist, ist man im allgemeinen an den granulierten Zellen ohne besondere Beachtung vorbeigegangen. Mán hat sich im wesentlichen begnügt, ihr Vorhandensein festzustellen, ohne aber auf ihre nähere Genese einzugehen. Speziell beim Karzinom hat man lediglich die eosinophil granulierten Leukozyten berücksichtigt, die basophilen Elemente sind nur von wenigen Autoren (Bonney 1907, 1908, Fromme) untersucht worden.

Es erschien uns deshalb als eine lohnende Aufgabe, zu diesem Zwecke frisch exzidiertes Karzinomgewebe einer Untersuchung zu unterziehen. Das Ziel, welches wir dabei verfolgten, war weniger das Studium der Wechselbeziehungen zwischen

1) Vergl. zur theoretischen Begründung: G. Ricker, Grundlinien einer Logik der Physiologie als reiner Naturwissenschaft. Jena, Gustav Fischer, 1912. 
Karzinomgewebe und dem Auftreten von granulierten Leukozytenformen äberhaupt, als vielmehr das Vorkommen dieser Elemente vom rein morphologischen Standpunkte aus zu beschreiben. Etwaige genetische Zusammenhänge zwischen ungranulierten und granulierten Leukozyten im Karzinomgewebe sollten klargelegt werden: So liefern wir damit einen Beitrag zu dem interessanten und noch viel umstrittenen Gebiet der Entstehung weißer Blutkörperehen auf ,monistischer" oder ,dualistischer" Grundlage.

\section{Material und Untersuchungsmethoden.}

Das Material entstammt zwei ulzerierten Plattenepithelkarzinomen der Portio einer 62 jührigen (Frau Sch.) und einer 39 jährigen (Frau P.). Die Stückchen wurden exzidiert und sofort in Zenker-Formalin (Modifikation nach Helly, 93 Zenker - und 7 Formalin) bei $37^{\circ}$ auf 2 Stunden gebracht. Es folgte die übliche Behandlung: Wässern, Härten, Einbetten in Paraffin. Die Schnittdicke betrug $4-6 \mu$.

Von Färbungsmethoden wurden angewandt:

1. Hämatoxylin-Eosin. Sie bringt sehr gute Übersichtsbilder hervor, vor allem zeigt sie übersichtlich die Verteilung von Karzinom und Bindegewebe. Die eosinophilen Zellen sind im allgemeinen sehr deutlich zu erkennen.

2. Giemsa-Romanowsky-Färbung in Schriddeseher Modifikation. Es wurden 2 Tropfen Farblösung auf $1 \mathrm{ccm}$ aq. dest. gebraucht. Die Färbungsdauer betrug 20 Minuten. Nach kurzem Abspülen folgte Entwässern in Aceton. puriss., Einschließen in Xylol und Kanadabalsam. Mit dieser Methode wurden dre vorzüglichsten Bilder hervorgebracht. Kern- und Plasmafärbung wie auch Darstellung der Granula gelangen taciellos.

3. Methylgrun-Pyroninfärbung nach Pappenheim. 35 Teile einer $1 \%$-Pyroninlösung werden mit 15 Teilen einer $1 \%$-Methylgrünlösung frisch zusammengegossen. Die Färbungsdaner beträgt 3 Minuten. Die Farbe wird in Wasser kurz abgespuilt, das Präparat rasch in Azeton differenziert und in Kanadabalsam eingeschlossen. Die Methode ist vor allem zur Darstellung der Plasmazellen und Mastzellen geeignet.

\section{Literatur.}

E. Schwarz hat in seinem ausführlichen Referat über die ,Lehre von der allgemeinen und örtlichen Eosinophilie" auch eine ersehöpfende Darstellung der Verhältnisse beim Karzinom gegeben. Es erübrigt sich daher, das bisher über diesen Gegenstand Veröffentlichte noch einmal in allen Einzelheiten zu wiederholèn. Fis sei an dieser. Stelle nur das Wichtigste hervorgehoben.

Unter 8 Portiokarzinomen findet B. Lewy 4 mit spärlichen eosinophilen Zellen, die anderen 4 enthielten diese in reichem Maße. „Lange Züge eosinophiler Zellen schoben sich zwischen die Bindegewebsfibrillen, ja auch zwischen die der Portio angehörenden Bündel glatter Muskelfasern hinein." Über ihre Kernmorpho- 
logie wird niehts erwähnt. Den gleichen Befund erhebt Noesske, welcher in dent Auftreten der Eosinophilen eine Reaktion des Organismus im Sinne der Abwehr entzündlicher Stoffe erblickt. Nach Feldbausch kommen sie im Karzinom zahlreich vor, jedoch sind sie von außen eingewandert; für ihre lokale Entstehung läßt sich kein Anhaltspunkt finden. Uninukleäre eosinophile Zellen werden von Cullen im Karzinom festgestellt, er läBt sie aber, ,undoubtedly" aus den Gefäßen aus wandern.

Bei ulzerierten Krebsen sind sie überaus reichlich zwischen den Epithelzellen vorhanden (Bonney), weisen aber alle multinukleäre Form auf. . Nach den zahlreichen Beobachtungen von Schottländer und Kermanner an Uteruskarzinomen wechselt ihre Zahl in den einzelnen Fällen erheblich. Meistens sind Kompaktkernige und Polymorphkernige gemischt, keinesfalls überwiegen die ersteren. Nach Ansicht der genannten Autoren spricht nichts für eine lokale Entstehung innerhalb des Karzinomgewebes. Nicht dortselbst, aber auch nicht im Knochenmark, wie man dann annehmen sollte, entstehen sie nach Nádosy, sondern in den regionären Lymphdrüsen. Das Fehlen von Mitosen und Myeloblasten, ihr sehr reichliches Vorkommen immerhalb der Blutgefäße ist der Grund, warum Fischer keine lokale Entstehung der eosinophilen Zellen annehmen kann. Dagegen wendet sich Weishaupt, welche behauptet, daß das Vorhandensein eosinophiler Leukozyten in den Blutgefaßen nicht in allen Fallen für eime Auswanderung aus denselben spreche. Es kann auch gleichzeitig lokal gebildete Eosinophilie init genereller Bluteosinophilie (Knochenmarkseosinophilie) bestehen (Pappenheim).

Die Zahl der eosinophilen Zellen im Blute bei Karzinomen erfuhr nach Przewoski keine Verändering, weder im. Sinne einer Vermehrung, noch einer Verminderung, noch in ihrem quantitativen Verhältnis zu den übrigen Lenkozyten. Voßwinkel findet kein gesetzmäßiges Verhalten der eosinophilen Leukozyten im Blute, ebenso Baradulin.

Die Plasmazellen im entzündlichen Infiltrate bei Karzinomen sind ron Sornani zum Gegenstand einer Untersuchung gemacht worden. Fr leitet sie von Lymphozyten ab. Zum gleichen Ergebnis kommen Schottländer und Kermauner. Diese Autoren haben bei Uteruskarzinom die Neubildung von Lymphknötchen beobachtet, wie sie auch von Ribbert und Bonney beschrieben sind. Letzterer sieht ebenfalls die kleinen Lymphozyten als die Mutterformen der Plasmazellen an.

Die Mastzellen kommen nach Bonney (1907) ziemlich selten vor, später firdet er sie aber ofter (1908). Fromme findet in den regionären Lymphdrüsenui die noch nicht bakteriell infiziert oder karzinomatös entartet sind, eine starke Anhäufung von Mastzellen. Dagegen ist ihre Zahl in den karzinomatösen oder infizierten Drüsen erheblich vermindert. Fromme glaubt daher annelimen zu müssen, daß sie toxische Sabstanzen vernichten, bzw. beim Aufbau von Bindegewebe in den Druisen eine Rolle spielen. 


\section{Befundbeschreibung.}

a) Eosinophile Zellen. Die Zahl der Zellen, deren Protoplasma nach Hämalaun-Eosin- oder Giemsa-Färbung eosinophile Körnchen aufweist, ist eine sehr wechselnde. Oft findet man sie in einem Schnitt zu vielen Exemplaren reihenweise angeordnet, besonders zwisehen den einzelnen Bündeln glatter Muskelfasern der Muskularis. Sonst ist ihr Vorkommen mehr vereinzelt, auBer an der angegebenen Stelle trifft man eine richtige Herdbildung nirgends. Wie wir weiter unten sehen werden, ist ihre Verteilung in Form von Strängen an eine bestimmte Kernkonfiguration gebunden. Vereinzelt liegen sie in der Hauptsache in dem bindegewebigen Stroma des Karzinoms (Fig. 1 u. 2, Taf. IV) inmitten der zelligen Infliltration. Jedoch kommen sie auch zwischen den Epithelzollen der Geschwulst nicht selten vor (Fig. 3, Taf. IV), aber ausschließlich dann, wenn die mehr oder weniger solide Verbindung der Karzinomzellen sich gelöst hat. Sodann zeigt Fig. 4, Taf. IV, daß sie auch in nicht krebsig entartetem Gewebe anzutreffen sind. Das Bild entstammt. der nächsten Umgebung des Portiokarzinoms, aber schon beim Utbergang in dein mit Zylinderepithel ausgekleideten Teil der Zervix. Daß die eosinophilen Zellen an die Umgebung der Blutgefäße gebunden sind oder in ihrer Nähe sich besonders reichlich zeigten, konnten wir nicht bemerken. Vielmehr lieb sich gar keine allgemein guiltige Regel über ihr Vorhandensein aufstellen. Nur an einer Stelle fehlten sie ganz, nämlich đa, wo sekundär eitrige Prozesse auftraten und das ganze Gewebe von spezialgranulierten Leukozyten durchsetzt war. Unter den vielen Tausenden von Zellen, die ein solches eitriges Exsudat aufbauen, fand sich niemals auch nur eine einzige eosinophile.

Diese Zellen sind im allgemeinen runde Gebilde (Fig. 1 emy ${ }_{1}$, Fig. 2 emy, Taf. IV), zeigen aber sehr oft Fortaatzbildungen (Fig. $3 \mathrm{emy}$, Taf. IV), ein Beweis amöboider Beweglichkeit. Je nach ihrer Lagerung im Gewebe sind sie auch oval oder aber langgestreckt (Fig. $1 \mathrm{emy}_{2}$, Taf. IV). Besonders bei größeren Ansammlungen eosinophiler Zellen, wie in den oben erwähnten Räumen zwischen den Muskelbündeln, ist ihre Gestalt schmal und lang, oft so schmal, daß die Zelle nur aus einem einzigen Protoplasmafaden zu bestehen scheint.

Das Plasma weist in seiner Färbbarkeit keine Besonderheit auf: es färbt sich leicht basophil. Der ganze Zelleib ist erfüllt von Körnern, welche sich mit Eosin intensiv rot färben. Die Granula sind ziemlich grob, unter sich gleich grob, von runder Gestalt und erfüllen die Zelle in gleichmäBig dichter Verteilung. In der Nähe des Kernes bleibt bei guter Fixation und Färbung eine Stelle von Granulationen frei, der Sitz des Mikrozentrums.

Interessant ist die Kernform dieser Elemente. Der Kern ist meist exzentrisch gelagert und weist kompakte oder gelappte Gestalt auf. Die kompaktkernigen Zellen besitzen große runde (Fig. $1 \mathrm{emy}_{3}$, Fig. $3 \mathrm{emy}$, Fig. 4 emy, Taf. IV) oder ovale (Fig. 2 emy, Taf. IV) Kerne. Die ganze Kernmasse färbt sich nicht wesentlich, das Chromatin ist unregelmäßig in Form feiner Fäden, Bröckel und Balken verteilt. Fine zweite Art von Kernen ist bedeutend kleiner als die vorher be- 
sprochene (Fig: $1 \mathrm{emy}_{2}$, Taf. IV). So häufig wie die voluminösen Kerne sind sie nicht aufzufinden. Die Grundfärbung dieses kleineren Kernes ist viel dunkler. Sein Chromatin weist die Form von gröberen Sehollen auf. Finige Male aber, doch ziemlich selten, finden wir typische ,Radkernstruktur". Zwischen diesen beiden Kernarten lassen sich alle Übergänge, sowohl in bezug auf ihre Größe wie die Färbbarkeit und Verteilung des Chromatins feststellen. Wir werden später noch auf ihre Bedeutung einzugehen haben. Die eben beschriebenen Zellen mit ihren ,kompakten“, kugeligen Kernen bilden den Übergang zu solchen Elementen, deren Kerne eine mehr oder weniger tiefe Einbuchtung aufweisen, also Nierenoder Hantelform besitzen, letztere wieder zu Zellen mit polymorphen Kernen (Fig. 3 el, Taf. IV). Aus letzteren setzen sich ausschließlich die oben erwähnten größeren Ansammlungen zwischen den einzelnen Muskelbündeln zusammen. Alle Zellen eosinophiler Natur besitzen rubende Kernform; mitotische Teilungsfiguren haben wir nicht feststellen können.

Die Natur dieser eosinophilen Elemente kann nicht zweifelhaft sein. Betrachtet man zunäehst die zuletzt erwähnten gelapptkernigen Zellen, so ergibt ein Vergleich mit den Blutelementen, daß sie identisch mit den gewöhnlichen eosinophilen Leukozyten des strömenden Blutes sind, denn außer dem Kern sind auch die Granula in ihrer Größe und Färbbarkeit absolut mit denjenigen Formen übereinstimmend, die sonst im Blute oder auch im Gewebe angetroffen werden. Von den polymorphkernigen Zellen bestehen alle Übergänge $\mathrm{zu}$ den kompaktkernigen, letztere aber stimmen vollständig in allen ihren Merkmalen überein mit den uninukleären eosinophilen Leukozyten des Knochenmarks, den Myelozyten. Besonders gilt das für die großkernigen Formen (Fig. 3 emy, Fig. 1 emy 3 , Taf. IV). Auf die dunkelkernigen Zellen kommen wir später noch zurück.

Es ergibt sich also, dab die eosinophilen Zellen des Karzinoms typische Leukozyten sind, daßsie entweder den polymorphkernigen Blutelementen oder aber den kompaktkernigen Myelozyten des Knochenmarks entsprechen und gleichzusetzen sind.

b) Neutrophile Leukozyten. Das Vorkommen neutrophil-granuliertex Elemente beschränkt sich auf diejenigen Stellen des Karzinoms, wo eine entzündliche Exsudation eingesetzt hat. Daselbst bestehen sämtliche Formen aus typischen "Eiterzellen". Ganz im Gegensatz zu den eben besprochenen Eosinophilen sind sie nie mit lymphozytären Typen untermischt. Streng genommen, gehören sie nicht zu den charakteristischen Finlagerungen des urspriinglichen Bindegewebes im Karzinom, sondern sind als sekundäre Erscheinungen aufzufassen. Somit scheiden sie für die vorliegende Betrachtung aus.

c) Vastzellen. Basophilgranulierte Elemente stellen in dem untersuchten Karzinomgewebe einen sehr häufigen Befund dar. Man trifft diese Formen ausschließlich in dem bindegewebigen Teil der Schnitte. Im epithelialen Gewebe fehlen sie vollständig. Sie sind nicht wie die anderen granulierten Zellen oft zu Gruppen oder Herden vereint, sondern liegen immer nur vereinzelt inmitten der 
iubrigen. Im ausgebildeten Zustand bilden sie langgestrekte, plumpe Elemente ohne wesentliche Fortsatzbildungen. Protoplasma ist bei den mit Körnern ganz erfüllten Zellen nicht zu sehen. Da wo die geringere Anzahl und weniger dichte Lagerung der Körnchen es zum Vorschein kommen läßt, erscheint es leicht basophil oder metachromatisch. Die Granula dieser Zellen färben sich mit MethylgrünPyronin-Misehung leuchtend rot (Fig. $5 m z_{1}, m z_{2}$, Taf. IV). Nach GiemsaFärbung erseheinen sie leicht hellblau bis tief dunkelblan (Fig. 2 ma, Taf. IV) oder aber dunkelblauviolett (Fig. $6 \mathrm{mz}$, Taf. IV), sind also basophil und deutlich metachromatisch. Bei Färbung mit Hämalaun-Eosin zeigen einzelne dieser Zellen eine ganz leichte, aber sehr deutliche Azidophilie (Fig. $4 \mathrm{mz}$, Taf. IV), auf Grund deren man sie auf den ersten Blick sehr leicht mit eosinophilen Zellen verwechseln kann.

Die Körnchen erfüllen den Zelleib so dicht, daß der Kern oft völlig von ihnen verdeckt wird. Ihre Größe und Gestalt wechseln sehr. Oft trifft man in einer Zelle neben ganz feinen staubförmigen Finlagerungen grobe Körner, die an Volumen den Granulationen der eosinophilen Zellen gleichkommen oder sie übertreffen. Aueh ihre Verteilung innerhalb ein und derselben Zelle weehselt mit ibrer Größe.

Neben den beschriebenen ausgebildeten Zellarten trifft man, wenn auch nicht so häufig, solehe, die viel spärlieher Granula enthalten. Die.Körnung beschränkt sich in ihrer Lage bei solchen Zellen meist auf die Rindenzone des Protoplasmas. Die Kerne dieser Typen färben sich metachromatisch oder basophil. Sie liegen meist zentral'(Fig. $5 m z_{1}, m z_{2}$, Taf. IV). Thre Form ist rund oder längsoval (Fig. 2 $m z$, Taf. IV), seltener leicht eingebuchtet (Fig. $4 \mathrm{mz}$, Fig. $6 \mathrm{mz}$, Taf. IV), aber nie gelappt. Meistens ist der Kern in seiner Grundsubstanz hell gefärbt, das Chromatin ungleichmäßig in Form feiner Bröckel und Balken in ihm verteilt (Fig. 6 mz, Fig. 4 mz, Taf. IV). Die ganze Struktur kann aber auch, besonders in den mehr langgestreckten Formen, verwischt sein (Fig. 2 mz, Taf. IV). Typische kleinkernige Elemente mit „Radkern" sind ein verhältnismäßig seltenes Vorkommnis und auch nur bei ganz wenig granulierten Formen anzutreffen. Mitosen konnten wir in diesen Zellen nie feststellen.

Die eben beschriebenen Formen stellen Mastzellen dar, wie man sie überall im Bindegewebe des Mensehen auffinden kann, Mastzellen in fertig ausgebildetem Zustand wie auch solche, die erst in der Fntwicklung begriffen sind.

d) Plasmazellen. Wir kommen zu der Beschreibung der ungranulierten Zellformen. An ihnen haben diejenigen Flemente einen wesentlichen Anteil, welche sich dureh eine starke Basophilie ihres reichlich entwickelten Plasmas und eine durchgehend gleichartige Kernkonfiguration auszeichnen. Überall im bindegewebigen Anteil des Karzinoms sind sie bald in dichterer Anordnung, bald vereinzelt anzutreffen. Ihre Form ist im wesentlichen rund oder leicht oval; längere Fortsatzbildungen fehlen. Das Protoplasma erseheint nach Giemsa-Färbung intensiv blau bis blauviolett (Fig. 7 pz, Taf. IV), seine Struktur ist mehr oder weniger homogen. Die gleichartige Besehaffenheit des ganzen Plasmaleibes ist in 
Fig. $7 p z$, Taf. IV deutlich zu erkennen, dagegen ist das Protoplasma in der anderem Zelle (Fig. $7 p z_{1}$, Taf. IV) nach dem Rande zu stark schollig aufgelockert. Fig. $7 p z_{2}$, Taf. IV zeigt diese Auflockerung im Bereich der ganzen Zelle. Die Pappenheimsche Methylgrün-Pyronin-Mischung erzeugt einen tiefroten Zelleib (Fig. 5 pz, Taf. IV). Jedoch kommen die feineren Auflockerungen des Protoplasmas bei Färbung nach Giemsa viel besser zur Darstellung.

Das Protoplasma weist durchgehend noch eine Besonderheit auf. Neben dem Kern liegt nämlich fast immer ein durch seine hellere Färbung und scharfe $\mathrm{Ab}$ grenzung mehr oder weniger deutlich erkennbarer Hof, die juxtanukleäre Vakuole.

Die Kernform dieser Elemente ist klein, gewöhnlich rund (Fig. 5 pz, Fig. 7 pz, Taf. IV), oft auch leicht oval (Fig. $5 z_{1}$, Taf. IV). In den größeren Zellen liegt der Kern exzentriseh (Fig. $7 p z_{1}, p z_{2}$, Taf. IV), in den kleineren gewöhnlich zentral (Fig. 5 p $z_{2}$, Taf. IV). Die Struktur des Kernes ist fast stets eine ausgesprochene "Radstruktur". Die soeben beschriebenen Formen sind als Plasmazellen von Marschalkóschem Typus zu bezeichnen. Außer diesen Zellen ausgeprägten Charakters trifft man aber sehr oft noch solche, die eines der vorgenannten Merkmale nicht so deutlich zeigen. So besitzt $z$. B. der in Fig. $5 z_{3}$, Taf. IV dargestellte Zelltypus dunkles, stark basophiles Plasma, das aber nicht sehr reichlich ausgebildet ist. Eine Vakuole ist nicht vorhanden, die Radstruktur des Kernes angedeutet. Derart ausgebildete Formen bilden den Übergang zu den gleich zu besprechenden lymphozytären Typen.

Eis wurde oben schon darauf hingewiesen, daß in manchen dieser Plasmazellen der Zelleib verwaschen granuliert erscheint. Gerade in der nächsten Umgebung solcher Formen, welche zudem noch kurze und gedrängte Ausläufer aufweisen, finden sich kleine protoplasmatische Abschnürungen. Sie lösen sich rom Ziellande als kugelige Gebilde von ungleicher Größe ab (Fig. $7 / p$, Taf. IV). Thre Färbung erscheint entsprechend ihrer Mutterzelle tief basophil. Sie liegen frei zwischen den einzelnen Elementen, oft als Anhäufungen einer Anzahl von kleinern Kugeln (Fig. 7 fp, Taf. IV), oft aber auch vereinzelt in Form von größeren Protoplasmaklumpen (Fig. 7 fp ${ }_{1}$, Fig. 5 tp ${ }_{1}$, Taf. IV).

e) Lymphozyten. Die überwiegende Mehrheit im bindegewebigen Stroma des Karzinomgewebes bilden ungranulierte Filemente von runder, oft auch leicht ovaler Gestalt. Sie liegen in dichten Haufen zwischen den einzelnen Bindegewebszügen, ohne daß eine scharfe Begrenzung gegenüber dem epithelialen bzw. muskulären Gewebe stattfindet. In allen Zwischenräumen des Bindegewebes sind gie vereinzelt anzutreffen.

Die Zellformen sind charakterisiert neben ihrer oben erwähnten runden bzw. ovalen Form durch ein mehr oder weniger reichlich entwickeltes Protoplasma, welches eine geringgradige Basophilie erkennen läbt. Finlagerungen irgendweleher Art oder schollige Struktur ist nirgends nachweisbar, vielmehr erscheint es überall ganz homogen. Bei solehen Typen, bei denen es spärlich entwickelt ist, umgibt es den Kern allseitig in Form eines schmalen Saumes. Andere Zellen weisen eine 
reichlichere Ausbildung des Protoplasmas auf (Fig. $1 l_{2}$, Taf. IV), bei diesen ganz groben ist der Plasmamantel nicht überall gleich breit. Letztere treten an Zahl bedeutend hinter den kleineren zurïck.

Der Kern dieser Elemente ist rund, im Verhältnis zu dem schmalen Protoplasmasaum sehr groß (Fig. $1 l_{1}$, Fig. $6 l_{1}$, Taf. IV). Das Chromatin färbt sich tief basophil und liegt in ihm in mehr odęr weniger regelmäßiger Anordnung zerstreut. Es weist in typischer Ausbildung ,Radstruktur" auf (Fig. $6 l_{2}$, Taf. IV). Andererseits findet man neben diesen verhältnismäßig großkernigen, aber im ganzen doch ,kleinen" Zelltypen Elemente mit einem viel voluminöseren, runden (Fig. $1 l y_{2}$, Taf. IV), ovalen (Fig. $1 y_{3}$, Taf. IV), leicht oder tief eingebuchteten Kern. Fr färbt sich wenig intensiv. Sein Chromatin ist in Form von feinen Fäden, Brocken und Balken in unregelmäßiger Weise in ihm verteilt. Nukleolen sind nicht selten.

Zwischen diesen extremen Ausbildungsformen der kleinen Zelle mit dem "Radkern" und der größeren protoplasmareichen finden sich sowohl in bezug auf Größe oder Gestalt des Kernes als auch die mehr oder weniger reichlichere Ausbildung des Plasmas sämtliche Übergänge. Nitotische Kernteilungsfiguren sind nicht selten; jedoch kommt es nicht, soweit wir feststellen konnten, zur Ausbildung typischer Keimzentren.

Vorstehend beschriebene Elemente sind als ,kleine" bzw. „gro Be": Lymphozyten mit allen' ihren charakteristischen Merkmalen aufzufassen.

Sie beherrsehen den bindegewebigen Anteil des Karzinomgewebes, in dem sich, an Zahl nicht wesentlich hinter ihnen zurückstehend und mit ihnen durehmiseht, zunächst die Plasmazellen, dann aber die vorstehend besprochenen granulierten Elemente finden.

f) Russelsche Körperchen. Man trifft ziemlich selten große Elemente, welche in ihrem ganzen Aufbau sich von den bisher besehriebenen wesentlich unterseheiden. Nicht nur, daß sie ihrem Volumen nach viel größer als die übrigen Formen (Fig. $7 w$, Taf. IV) sind, ihr Protoplasma ist von ungleich großen kugelförmigen Gebilden vollständig ausgefüllt. Die abgebildete Zelle gibt einen solchen Typus wieder. Das Plasma besteht aus jenen Kugeln selbst, welche mit einer flüssigen, in der Mitte jeder Kugel heller erseheinenden Masse gefüllt sind, sich nach Giemsa-Färbung in einen lila Ton tingieren. Daneben gibt es aber auch andere Formen, in denen sich diese „Vakuolen". riel dunkler blau, in dem Tone des Plasmas der Plasmazellen, färben, oder aber nach Hämatoxylin-Eosinfärbung in einem blassen Rot, etwa in dem Tone der in Fig. $4 m z$, Taf. IV dargestellten Mastzelle. Die Zahl der Vakuolen innerhalb der Zelle ist eine wechselnde, sie schwankt zwischen 4 oder 5 größeren Hohlräumen oder aber einer erheblicheren Anzahl weniger voluminöser Kugeln (Fig. $7 r k$, Taf. IV). Gerade bei den größere Kugeln enthaltenden Zellen findet sehr oft eine gegenseitige Abplattung dieser Hohlräume statt, ohne daß sich aber ein Zusammenfließen des einen mit dem anderen nachweisen läßt. 
Der Kern weist wechselnde Gestalt, immer aber starke Färbung seines Chromatins auf; er liegt stets exzentriseh am äußeren Rande der Zelle. Seine Grundform ist rund, mit tiefen oder flachen, durch die benachbarten Vakuolen bedingten Einbuchtungen; oft ist er als ganz schmaler langgestreckter Chromatinstreifen ohne deutlich erkennbare Struktur am Rande der Zelle sichtbar. Da, wo er noch nicht zu viel eingeengt ist, erkennt man in ihm eine mehr oder minder ausgesprochene ,Radstruktur".

Diese Zellformen sind als die schon oft beschriebenen, zuerst von Russel in Karzinomgewebe gefundenen "Fuchsinkörperchen" anzusehen. Sie lassen sich im vorliegenden Gewebe in allen ihren Entwicklungsstufen von der in Fig. $7 \pi \%$, Taf. IV bildlich dargestellten' Form bis zu den Elementen verfolgen, welche als Plasmazellen mit vakuolisiertem Protoplasma anzusehen sind. Fin Zusammenhang mit letzteren, der an anderer Stelle ausführlich dargestellt werden soll, kann nicht zweifelhaft sein.

\section{Kritische Erörterung.}

Wir haben in den vorstehenden Zeilen die granulierten sowohl wie die ungranulierten Formen des lymphoiden Gewebes im bindegewebigen Teil des Karzinoms aul Grund unserer Befunde dargestellt. Für die eosinophilen Zellen konnten wir nachweisen, daß es sich um typische azidophile Leukozyten handelt, wie sie auch ron anderen Autoren (Lewy, Noesske, Feldbausch, Bonney, Schottländer und Kermauner, Fischer, Weishaupt) gefunden wurden. Für ihren Charakter als mit den Blutelementen identischen Zellen spricht das Aussehen ihrer Granula, mehr aber noch das Verhalten ihrer Kerne. Von der polymorphkernigen über die zwerchsack- und hantelkernige Form bis zu der kompaktkernigen Zelle lassen sich alle Übergangsstufen auffinden. Also besteht nicht nur die Sicherheit der vollen Identität der eosinophil granulierten Zellen mit der entspreehenden Lenkozytenform des Blutes, sondern es ergibt sich aus dem Nachweis der ,Kompaktkernigen" im Karzinomgewebe, daß wie im leukopoetischen Gewebe anch im bindegewebigen Anteil des Krebses eine Tmformung von polymorphkernigen aus kompaktkernigen Formen stattfindet.

Aus unseren Abbildungen geht einwandfrei hervor, daß wir in den wiedergegebenen Zellformen Elemente uninukleärer Natur vor uns haben. Unsere Untersuchung*en haben gezeigt, daß diese rundkernigen Zellen keineswegs einen seltenen Befund im Karzinomgewebe darstellen. Nach eingehenden Zählungen in zahlreichen Schnitten mußten wir die Zahl der uninukleären Formen auf etwa $25 \%$ annehmen, an einzelnen Stellen z. B. in dem Gewebsabschnitt, aus dem Fig. 4, Taf. IV entnommen ist, bilden sie die weitaus iberwiegende Mehrheit. Diese $\mathrm{Ab}-$ bildung stammt, wie wir schon bemerkten, aus dem Übergang des reinkarzinomatösen in den noch nich仑 durchwucherten Teil der Portio. Leider war uns Naterial zur Untersuchung an normalem Gewebe nicht zugänglich. Aber immerhin beweisen dic angegebenen Befunde, daß im Gegensatz zu den Angaben von Schottländer 
und Kermauner an einzelnen Stellen ein Überwiegen der Kompaktkernigen stattfindet.

Unsere Befunde sind zu eindeutig, als daß wir annehmen könnten, daß dureh die Schnittrichtung uninukleäre Formen vorgetäuscht werden, daß die andere Hälfte eines polymorphen Kernes weggeschnitten worden wäre. Übrigens haben wir uns bei der Durehsicht von Abstrichen dex Tumormasse davon überzeugt, daß dieselben kompaktkernigen Formen auch darin einwandfrei festzustellen sind.

Ehrlich und seine Sehule stehen auf dem Standpunkt, daß nur im Knochenmark ans cosinophilen Myelozyten durch Kernumformung gelapptkernige Leukozyten entstehen, daß eine Auswanderung von kompaktkernigen Formen aus dem Knochenmark im postembryonalen Leben nicht stattfindet. Wir selbst haben uns durch Blutuntersuchungen bei Lebzeiten der untersuchten Fälle vergewissert, daB zwar eosinophile Leukozyten, aber keine uninukleären Formen im Blute vorhanden sind. Die gefundenen Zahlen sehwanten zwischen $1,5 \%$ und $9 \%$ polymorphkernige Fosinophile. Die Blutbefunde beweisen also erstens, daß uninukleare Formen im Kreislauf nicht aufzufinden sind. Es muß daher angenommen werden, daß jene rundkernigen Zellen, welche wir im Vorstehenden als die Mutterform der polymorphen Zellen angenommen haben, an Ort und Stelle aus ungranulierten Zellen hervorgegangen sind. Zweitens geht aus den angegebenen Zahlen der Blutuntersuchungen hervor, daß Eosinophile nicht so reichlich im Blute kreisend getroffen werden, als daß man annehmen könnte, die Ansammlungen eosinophiler Leukozyten seien hämatogener Natur. Allerdings sind $9 \%$ Eosinophile rcichlich viel, doch haben wir unter unseren zahlreichen Untersuchungen diesen Befund nur einmal erheben können. Eine Teilung granulierter Elemente scheint nicht vor sich zu gehen, denn es fehlt uns der Nachweis von mitotisehen Figuren. Aber dennoch müssen wir auf Grund des Vorkommens uninukleärer Formen von Myelozyten, wie sie auch von Cullen, Schottländer und Kermauner gesehen wurden, eine autochthone Fintstehung innerhalb des Karzinomgewebes annehmen. Warum Cullen die eosinophilen Zellen ,undoubtedly" aus den GefäBen auswandern läßt, trotzdem ihm der Nachweis von Uninukleären gelungen ist, ist nicht verständlich. Daß auch in den regionären Lymphdrïsen, wie Nádosy meint, die Bildung der Polymorphkernigen vor sich geht, ist auf Grund unserer Befunde auch nicht anzunehmen.

Ebensowenig konnten wir ein sehr reichliches Vorkommen innerhalb der Blutgefäße (Fischer) feststellen und damit als ihren Entstehungsort das Knochenmark betrachten (Feldbausch). Daßs eine Auswanderung aus den Blutgefäßen stattfindet, ist zwar nicht unwahrscheinlich, aber naturgemäß nicht mit aller Sicherheit festzustellen. Bei der gesteigerten Emigration sämtlicher Arten weißer Blutkörperchen, besonders im Beginn der Infiltration, können auch die eosinophilen bis zu einem gewissen Grade beteiligt sein. Die Pappenheimsche Ansicht von einer gleichzeitigen lokalen Eosinophilie mit genereller Bluteosinophilie (Knochenmarks-Eosinophilie) scheint jedoch auf den vorliegenden Fall nicht 
zuzutreffen, weil, wie oben schon gesagt, eine generelle Bluteosinophilie nicht nachweisbar ist.

Es ergibt sich also, daß innerhalb des bindegewebigen Anteils des Karzinomgewebes eine autochthone, lokale Entstehung von eosinophil granulierten Leukozyten aus ungranulierten Zellen stattfindet. Über die Abstammung der letzteren werden wir gleich zu berichten haben.

Das Auftreten neutrophil-granulierter Leukozyten ist, wie in der Befundbeschreibung hervorgehoben, auf sekundär entzuindliche Prozesse zurückzuführen. Wir finden deshalb in dem untersuchten Gewebe im allgemeinen diese Elemente nicht, nur an einzelnen Stellen kommt es za gröBeren Ansammlungen, welcheaber dann ausschließlich sich aus solchen zusammensetzen. Die pyknotische Natur ihrer Kerne, die sehlechte Färbbarkeit ihrer Granula charakterisieren sie von vornherein als ,Eiterzellen" ${ }^{\text {tc }}$ und damit als aus den Blutgefäßen ausgewanderte Elemente. Für die vorliegenden Untersuchungen kommen sie deshalb nicht in Betracht.

Die Mastzellen bilden, das beweisen unsere Befunde, ein häufiges Vorkommnis. Sie finden sich im Bindegewebe ïberall zerstreut. Beziehungen zum Blute haben sie keine, da ,histogene Mastzellen", wie wir sie hier vor uns haben, im Blute nicht zu finden sind. Sie besitzen im allgemeinen keine gelappte Kernform. Fis läßt sich also hier nicht wie bei den anderen granulierten Elementen durch den Nachweis der Kernumformung ihre Entstehung aus rundkernigen Elementen in loco verfolgen. Dagegen konnten wir Zellformen auffinden, welche ganz den Charakter von Plasmazellen bzw. kleinen Lymphozyten aufwiesen, nur daß eben die Randzone des Protoplasmas mit mehr oder weniger feinen basophilen Körnchen durchsetzt ist. Mitosen solcher Formen sind nicht festzustellen. Es muß also eine direkte Umwandlung von Ungranulierten vom Typus der kleinen Lymphozyten und Plasmazellen in Mastzellen stattfinden.

Die Plasmazellen stellen einen konstanten Bestandteil des lymphoiden Gewebes dar. Wir verstehen darunter nicht nur die Zellen vom rein Marschalkóschen Typus, sondern auch alle jene, welche durch ihre Kernbeschaffenheit den verschiedenartigen Formen des lymphoiden Gewebes nahestehen und deshalb nach Weidenreich (1909) infolge der Beschaffenheit ihres Protoplasmas auch als Plasmazellen zu betrachten sind. Wir haben alle möglichen Übergänge zwischen Plasmazellen und gewöhnlichen Lymphozyten festgestellt und erblicken deshalb mit Sornani, Schottländer und Kermauner, Bonney in den kleinen Lymphozyten die Mutterformen der Plasmazellen.

Die Russelschen Körperchen zeigen in den jüngeren Ausbildungsstadien, d. h. in denjenigen Formen, bei welehen sich das Protoplasma aus einigen ganz großen Vakuolen zusammensetzt, wo vor allem der Kern mehr oder weniger ausgeprägte Radstruktur aufweist, weitgehende Beziehungen zu den Plasmazellen. Es kann kein Zweifel bestehen, dab sie aus solchen Typen hervorgehen, wie wir sie in Fig. $7 p z_{1}, p z_{2}$, Taf. IV beschrieben haben, wo das Protoplasma eine sehollige Struktur verbunden mit leichter Azidophilie zeigt. Ob die Ausbildung der Russel- 
schen Körperchen als hyaline Degeneration, oder wie sie anders aufgefart werden soll, wird an anderer Stelle ausfithrlich besprochen werden.

Die letzte der beschriebenen Zellformen bilden die Lymphozyten. Wir haben gesehen, daß sie den Hauptbestandteil der Wanderzellen innerhalb des Bindegewebes ausmachen. Neben kleinen und großen Elementen fanden wir sämtliche Übergänge zwischen beiden Typen, sowohl in bezug auf Größe, Gestalt, Bau des Kernes, als auch auf Färbbarkeit und Menge des Protoplasmas. Die Natur dieser Elemente als lymphoide Zellformen braucht wohl nicht weiter erörtert zu werden. Mitosen von Lymphozyten sind nicht selten; jedoch haben wir die Bildung von Keimzentren oder gar von Lymphknötchen, wie sie Sehottländer und Kermauner, Ribbert und Bonney beobachtet haben, nicht feststellen können.

Was die Herkunft der lymphoiden Flemente anbelangt, müssen wir annehmen, dab sie in dem chronischen Entzündungszustande, in dem sich das Untersuchungsmaterial befand, in der Hauptsache aus sich bereits im Bindegewebe vorhandenen Elementen lymphoider Natur ableiten lassen. Die ersten Lymphozyten sind, wie aus zahlreichen Studien aus der Entzündungslehre belannt ist, aus den Blutgefäßen ausgewanderte klein- und großkernige Elemente. Die Zellen der Adventitia spielen, wie neuerdings $u$, a. von Herzog hervorgehoben wird, auch eine große Rolle in den ersten Stadien der Entzündung. Hier ist nicht der Ort, auf diese Fragen der Herkunft der ersten Lymphozyten im entzündeten Gewebe einzugehen. Die in unseren Sehnitten nachweisbaren Zellformen ergänzen sich, wie gesagt, aus sich selbst heraus durch mitotische Teilung vor allem großkerniger Elemente. Auf jeden Fall spielt die Auswanderung aus den Blutgefäßen in den uns vorliegenden Stadien der Fintzündung eine untergeordnete Rolle. Denn die in den Schnitten getroffenen GefäßRe zeigen keinen besonderen Reichtum an lymphoiden Zellen. Die etwaigen Beziehungen zu den jungen Bindegewebæzellen, den Fibroblasten, können hier nicht näher erörtert werden.

\section{Die lokale Bildung der granulierten Zellen und die ubiquitäre Natur der Lymphozyten.}

Die Entstehung der granulierten Lenkozyten - beim Menschen also hauptsächlich die eosinophilen und neutrophilen Elemente - wird von Eihrlich und seinen Anhängern; von neueren Autoren, namentlich Schridde und Naegeli, unter normalen Verhältnissen ausschlieBlich in das Knochenmark verlegt. Dort gehen sie im postfötalen Leben entweder aus solchen Myelozyten hervor, welche sich durch mitotische Teilung bereits vorhandener ergänzen oder aber aus anderen, die aus einer nur im Knochenmark vorhandenen Zellform, den Myeloblasten, durch Ausarbeitung oder Aufnahme von Granulationen entstehen. Die aus dem Knochenmark ausgeschwemmten Formen, die ,polymorphkernigen " Lenkozyten, sind reife Elemente, welche einer weiteren Entwicklung sich nicht mehr fähig erweisen. Myelozyten kreisen unter normalen Verhältnissen im Blute nicht. Es 
kann daher außerhalb des Knochenmarks normalerweise eine lokale Entstehung granulierter Leukozyten nicht stattfinden. Wo man also solehe Formen in den Organen trifft, müssen sie aus den Blutgefäßen ausgewandert sein.

Demgemäß wird auch von den Anhängern der Ehrlichsehen Theorie, so von Fiseher, Sehottländer und Kermauner u. a., eine lokale Entstehung eosinophil-granulierter Elemente im Bindegewebe des Karzinoms - denn nur um die Eosinophilen handelt es sich im vorliegenden Falle - nicht zugegeben. Sehottländer und Kermauner beschreiben zwar das Vorkommen kompaktkerniger eosinophiler Zellen, aber es spricht nach ihnen trotzdem nichts für eine lokale Fintstehung innerhalb des Karzinomgewebes. Diesen Befunden gegenüber konnten wir nachweisen, daß man im Bindegewebe des Karzinoms zu $25 \%$ uninukleäre Formen findet. Daß es sich dabei um Flemente handelt, welche nieht nur morphologiseh, sondern auch funktionell mit den ,Myelozyten" der Ehrlich schen Nomenklatur übereinstimmen, geht daraus hervor, daß wir genau wie im Knochenmark sämtliche Übergänge von der rundkernigen bis zur gelapptkernigen Zelle feststellen konnten.

Woher stammen nun die eosinophilen Myelozyten im Karzinomgerrebe? Vom Standpunkte der Ehrlichschen Lehre, welche besagt, daßs im Blute nur polynorphkernige, einer weiteren Entwicklung unfähige Elemente kreisen, müßte man annehmen, daß diese Kompaktkernigen aus dem Knochenmark ausgeschwemmte Myelozyten sind, welche sich im Karzinomgewebe festgesetzt haben. Dagegen sprechen unsere Blutbefunde, aus denen hervorgeht, daß auch im Blute unserer Karzinomkranken keine uninukleären Formen vorkommen, dagegen sprechen auch die Blutuntersuchungen anderer Autoren (Baradulin, Przewoski, Vosswinkel) bei Karzinomatösen, wo der Nachweis von Myelozyten im strömenden Blute nicht geführt worden ist.

Da also als Entstehungsort der Myelozyten nicht das Knochenmark gelten kann, muß angenommen werden, daß sie autoehthon, lokal im Karzinom sich bilden. Mitotische Teilungsfiguren eosinophil granulierter Elemente waren, wie wir schon hervorgehoben haben, nicht nachzuweisen, sie müssen also aus ungranulierten Zellen hervorgegangen sein. Das stimmt überein mit der von Weidenreich, Pappenheim u. a. vertretenen Lehre, daß granulierte Leukozyten das ganze Leben hindurch aus ungranulierten Formen sich bilden, wie es auch Ehrlich, Naegeli und Schridde für das Knochenmark annehmen. Der Untersehied in der Auffassung von Ehrlich usw. und der von Weidenreich besteht aber đarin, daß Ehrlich für die granulierten Elemente besondere, eben nur im Knochenmark vorkommende Myeloblasten als Mutterformen gelten läßat, während die von Weidenreich vertretende Anschaung dahin geht, dab diese Myeloblasten den lymphozytären Eilementen im lymphoiden Gewebe gleichzusetzen sind. Wir konnten zeigen, daß im Karzinomgewebe neben den Myelozyten, also den granulierten, anch ungranulierte Formen (Fig. $1 y_{2}$, Taf. IV) vorhanden sind, welche morphologiseh durchaus den Myeloblasten entsprechen. Es ist also anzunehmen, 
da: $B$ aus diesen rein lymphoiden Formen durch Ausarbeitung oder Aufnahme von Granulationen die eosinophilen Myelozyten hervorgehen. Erkennt man diese Fähigkeit der extramedullären Bildung von granulierten aus ungranulierten Zellen den von uns als lymphoiden Elementen erkannten Zellen zu, so ist damit der Beweis für die ubiquitäre Natur der Elemente der lymphoiden. Zellreihe erbracht.

Die Möglichkeit, sich in eine granulierte Zelle umzuwandeln, ist nicht, wie angenommen wird, an eine bestimmte Ausbildungsform des Lymphozyten gebunden. Fin Blick auf unsere Abbildungen (Fig. $1 \mathrm{emy}_{1}, e m y_{2}, e m y_{3}$, Fig. $3 \mathrm{emy}$. Taf. IV) lehrt, da $\dot{B}$ nicht nur die grob- und hellkernigen Elemente der lymphoiden Reihe der Weiterentwicklung im Sinne einer Granulierung fähig sind, sondern daß auch die kieinen lymphoiden Formen sich in Granulozyten umbilden können. Fig. 3 emy, Taf. IV gibt eine Zelle wieder; welche dem Typus der großkernigen Wanderzelle zugehört, dagegen zeigt Fig. $1 \mathrm{emy}_{2}$, Taf. IV den dunkeln und ehromatinreichen Kern, wie ihn die kleineren lymphoiden Formen aufweisen. Diese Verschiedenartigkeit in der Kerngröße, Konfiguration und Färbbarkeit des Chromatins bildet einen zweiten Beweis dafür, daB sich aus ungranulierten Elementen granulierte Formen bilden können, welche keinen bestimmten Entwicklungszustand zur Voraussetzung haben; sie können ebensogut dem Typus des kleinen, mittelgroßen als auch des großen Lymphozyten angehören. „Die Fähigkeit, Granula im Plasma auszuarbeiten oder aufzunehmen, ist an keine bestimmte Erscheinungsform des Lymphozyten gebunden", sagt Weidenreich (11, S. 307). Das" Endprodukt, der eosinophile, polymorphkernige Leukozyt, ist in jedem Falle gleich, mag die Jutterform ein groß- oder ein kleinkerniger Lymphozyt gewesen sein. Man kann deshalb auch der Ausgangsform keine besondere Stellung zubilligen, besonders da auch die Myeloblasten im Knochenmark sowohl kleine als große Formen erkennen lassen, welche sich genau, wie wir für das Karzinomgewebe dargetan haben, in granulierte Zellen umwandeln. Es ergibt sich also auch daraus, daß der Lymphozyt diejenige Zellform ist, aus welcher sich im postfötalen Leben unter gegebenen Umständen der Granulozyt entwickeln kann und damit wiederum ein Beweis für den ubiquitären Charakter der lymphoiden Elemente.

Wir haben in diesem Zusanmenhang bisher nur von den eosinophilen Zellen und ihrer Entwicklung aus Lymphozyten gesprochen. Daß sich aber auch im. Karzinomgewebe noch andere Zellarten finden, welche dortselbst aus lymphoiden Typen ihren Ursprung nehmen, nämlich die Mast- und Plasmazellen, ist weiter vorn schon ausführlich besprochen worden. Der kleine Lymphozyt vergröBert seine Plasmamabe, indem gleichzeitig ihre Basophilie zunimmt und die juxtaunkleäre Vakuole deutlich wird. So bildet sich die Plasmazelle. Oder aber es entstehen durch Ausarbeitung basophiler Granula in der Randzone des Plasmas von Lymphozyten junge Mastzellen. Die Beziehungen zwischen lymphoiden Zellen and Plasmazellen werden heute allgemein anerkannt; dagegen herrscht über die Entstehung der Mastzellen noch keine einheitliche Auffassung. Soviel steht allerdings fest, daß die „Bindegewebsmastzellen" nicht im Knochenmark entstehen, sondern sich überall im Bindegewebe lokal bilden hönnen. 
Gs ist hier unmöglich and auch nicht nötig, alle die Beweise nochmals anzuführen, welche Weidenreich (11) ausführlich zusammengestellt hat, um klarzulegen, daß es gerade der "Lymphozyt" der Ehrlichschen Bezeichnung ist, welcher sich einer weitgehenden Differenzierung fähig erweist. Ein weiterer Beweis für die Richtigkeit dieser Ansehauung geht aus den vorliegenden Untersuchungen hervor. Auch unter pathologischen Verhältnissen ist die lymphoide Zelle imstande, außerhalb des Knochenmarks durch Aufnahme eosinophiler Körnchen sich in eine granulierte Form umzuwandeln. Andererseits findet in reichem Vaße eine Umbildung der lymphoiden in basophil granulierte Elemente oder aber in Plasmazellen statt.

Es wurde schon versehiedene Male hervorgehoben, daß von einer lokalen Entstehung neutrophiler Leukozyten im Karzinomgewebe nicht die Rede sein kann, sondern ausschließlich eine Entwicklung eosinophiler Zellen stattfindet. Es ist schwer zu sagen, womit diese auffallende Tatsache begründet werden kann. Es scheint, als ob die einseitige Differenzierung der Granulopoiese mit dem Austritt und Zerfall roter Blutkörperchen im Karzinom zusammenihängt. Kleinere und größere Blutmengen haben wir in unseren Schnitten öfters nachweisen können: doch sind uns freie eosinophile Granula nie begegnet.

$\mathrm{Ob}$ das Auftreten der eosinophilen Zellen mit der beginnenden Nebrose zusammenhängt, wie Noesske und Oehler meinen, ist nicht wahrscheinlich. Zwar entstammen unsere beiden Fälle stark exulzerierten Tumoren. Nimmt man als Folge der Exzulzeration eine bakterielle Infektion und damit eine neue Fntzündung an, so müßten sich mindestens zwischen den azidophilen Zellen auch solche mit neutrophilen Granulationen zeigen; das ist nun gar nicht der Fall. Vielmehr ist das Auftreten der Neutrophilen streng auf einzelne Stellen lokalisiert, da fehlen aber die $\alpha$-Zellen. Die Fintzündung scheint also die eosinophilen Zellen wieder aus dem Tumorgewebe zu vertreiben. Ob andererseits die Meinung von Feldbausch, daß, je weniger zerfallen ein Karzinom ist, um so mehr a-Zellen in ihn enthalten sind, die richtige ist, erscheint nach unseren Befunden zweifelhaft.

Ein positiver Anhaltspunkt dafür, daß die eosinoplastische Funktion des Bindegewebes im Karzinom auf ,einen spezifischen chemischen Reiz der Neubildung" (Schwarz, Fiseher) in Erscheinung tritt, ergibt sich aus unseren Untersuchungen nicht. Es kann hier nicht unsere Aufgabe sein, die ganze Literatur, welche für und gegen die eosinotaktisehe Funktion des Karzinomgewebes angeführt wird, auf ihre Richtigkeit zu untersuchen. Dafür fehlen uns auch die Grundlagen.

Wir beschränken uns auf unsere morphologisch sicherstehenden Befunde, aus dehnen hervorgeht, daB die Meinung von Schwarz, ,daß die Befunde bei Tumoren gar keinen Anhaltspunkt fuir lokale Genese der $\alpha$-Zellen geben" (S. 577), nicht richtig sein kann. Über die Griunde dieser nachgewiesenen lokalen Bildung eosinophiler Zellen uns weiter auszulassen, erscheint nicht zweckmäßig.

Es geht also aus unseren Beobachtungen hervor, dab auch unter pathologischen Verhältnissen, speziell im Karzinomgewebe vom Knochenmark unab- 
hängig aus lymphozytären Elementen eosinophile Leukozyten entstehen können. „Ganz abgesehen davon, dab die direkten Beziehungen zwischen Lymphozyten einerseits und diesen Lenkozyten andererseits noch an der charakteristischen Kernform und Struktur nachgewiesen werden können, läßt sich gerade für die unter pathologisehen Verhältnissen auftretende allgemeine oder lokale „Eosinophilie“ eine andere plausible Erklärung überhaupt nicht beibringen. Denn die Hypothese, daß irgend ein besonderer örtlicher Reiz auf das entfernte Knochenmark wirke, dort eine vermehrte Bildung jener Zellformen auslöse und die neugebildeten wieder in Maßen gerade nach jenem Gebiet hinführe, von dem dieser Reiz ausging, muß mit soviel Hilfshypothesen rechnen, daß schon ein starker Glaube dazu gehört, sie für wahrscheinlicher zu halten als die einfachere und in den meisten Fällen direkt beweisbare Annahme einer Entstehung an Ort und Stelle" (Weidenreich, 11, S. 306).

\section{Literatur.}

Baradulin, G. J., 1910, Über Blutveränderungen bei malignen Neubildungen. Fol. haemat. Bd. 9, S. 407-474. - Bonney, Y., 1907, A Study of the Connective Tissues in Squamous cells carcinoma and in certain pathological conditions preceeding its onset. Areh. of the Middlessex Hospital Yol. 9, S. 64. - Dersel be, 1908, The Connective Tissues in Carcinoma and in certain inflammatory States that preceede its onset. Lancet. vol.1,2. party, p. 1389, nr. 1465. - Cullen, T. S., 1900, Cancer of the Uterus. London. - Ehrlich, P. und Lazarns, A., 1898, Die Anämie. 1. Abt. Normale u. pathol. Histologie des Blutes. 1. Aufl. - Feld ba usch, F, 1900 , Über das Vorkommen von eosinophilen Leukozyten in Tumoren. Virch. Arch. Bd. 161, S. 1. Fis cher, W., 1912, Über die lokale Anhäufung eosinophil gekörnter Leukozyten in den Geweben, besonders beim Krebs. Tieglers Beiträge Bd. 55, S. 1. - Fromme, 1906, Demonstration über das Yerhalten der Mastzellen beim Karzinom. Ztlbl. f. Gynäkologie Bd. 30, S. 1146. - Herzog; G., 1915, Experimentelle Untersuchungen über die Einheilung von Frem dzörcem. Habil.-Schr., Jena 1915. - Lew y, B., 1900. Die Beziehungen der Charcot-Leyden schen Kristalle za den eosinophilen Zellen. Ztschr. f. klin. Med. Bd. 40, S. 59. - Nádosy, Die Drüsen beim Utenskarzinom. (Ungarisch.) Ref.: Ztlbl. f. Gynäkol. 1910, S. 29. - Noes ske, H., 1900, Eosinophile Zellen und Knochenmark, insbesondere bei chirurgischen Infektionskrankheiten und Geschwülsten. D. Ztschr. f. Chir. Bd. 55, S. 211. - Oehler, J., 1912, Beitrag zur Kenntnis der lokalen Eosinophilie bei chirurg. Darmaffektionen. Mitt. a. d. Grenzgeb. d. Med. u. Chirurg. Bd. 25, S. 568. Przewoski, E., 1896, Über die Jokale Eosinophilie beim Krebs nebst Bemerkungen uber die Bedeutung der eosinophilen Zellen im algemeinen. Ztlbl. f. allg. Pathol. Bd. 7, S. 177. - Russel, W., 1890 , An address on a characteristic organism of cancer. Brit. med. Journ. II, p. 1356. Schottländer, J. und Kermauner, F., 1912, Zur Kenntnis des Uteruskarzinoms. Berlin, S. Karger. - Sehwarz, E., 1914, Die Lehre von der allgemeinen und örtlichen ,Eosinophilie" Lubarsch a. Ostertag, Ergebn. d. allg. Pathol. Bd. 17, S. 1. - Sornani, B. P., 1906, Uber Plasmazellen in dem entzïndlichen Infiltrate eines Krebstumors des Magens. Virch. Arch. Bd. 184, S. 177. - Vo B winkel, K., 1898, Über das Vorkommen von eosinophilen Zellen und Myelozy ten im menschlichen Blute bei Erkrankung der inneren weiblichen Geschlechtsorgane. Mschr. f. Geburtshilfe u. Gyn. Bd. 7, S. 443. - Weidenreich, F., 1909, Zur Morphologie and morphologischen Stellung der ungranulierten Leukozyten - Lymphozyten des Blutes und der Lymphe. Arch. f. mikr. Anat. Bd. 73. - Derselbe, 1911, Die Leukozyten und verwandte Zellformen. Wiesbaden. - Weishaupt, E., 1913, Uber eosinophile Lenkozyten in entzündlichen Infittraten, besonders der mit und ohne Strahlentherapie vorbehandelten Uteruskarzinome. Areh. f. Gynälol, Bd. 101, H. 2. 


\section{Erklärung der Abbildungen auf Taf. IV.}

Unter "Material und Untersuchungsmethoden" ist im Text das Nähere über Fixation und Färbung der Präparate angegeben. Die Abkürzungen bedeuten: $G=$ Romanowsky-Färbung nach Giemsa, MP $=$ Färbung mit Methylgrün-Pyronin nach Pappenheim, HE = Färbung mit Hämalaun-Eosin.

Die Zeichnungen sind durchweg auf Objekttischhöhe aufgenommen, wo nicht anders vermerkt, mit: Zeib' Apochx. $1,5 \mathrm{~mm}$ and Okul 8 .

$e l=$ eosinophiler Leukozyt, $l y=$ Lymphozyt, em $y=$ eosynopiler Nyelozyt, $f p=$ protoplasmatische Abschnürung, $m z=$ Mastzelle, $p z=$ Plasmazelle, $r k=$ Russ el-Körper.

Fig. 1. Hërd von eosinophilen Myelozyten und verschiedener Typen von Lymphozyten aus dem Bindegewebe des Karzinoms der 62 jährigen. G.

Fig. 2. Myelozyt und Plasmazelle in Bindegewebe, 62jührige. G.

Fig. 3. Karzinomzellen mit eosinophylem Myelozyt und Leukozyt. 39jährige. G.

Fig. 4. Eosinophiler Myelozyt und Mastzelle aus dem Übergang des karzinomatösen in das gesunde Gervebe. 39 jährige. HE.

Fig. 5. Plasmazellen, Mastzellen und Plasmaabsehnürungen im Bindegewebe. 62jährige. MP.

Fig. 6. Mastzelle in Bíndegewebe. 62jährige. G.

Fig. 7. Plasmazellen und Russelsches Körperchen im Bindegewebe. 62jâhrige. G. 
sì
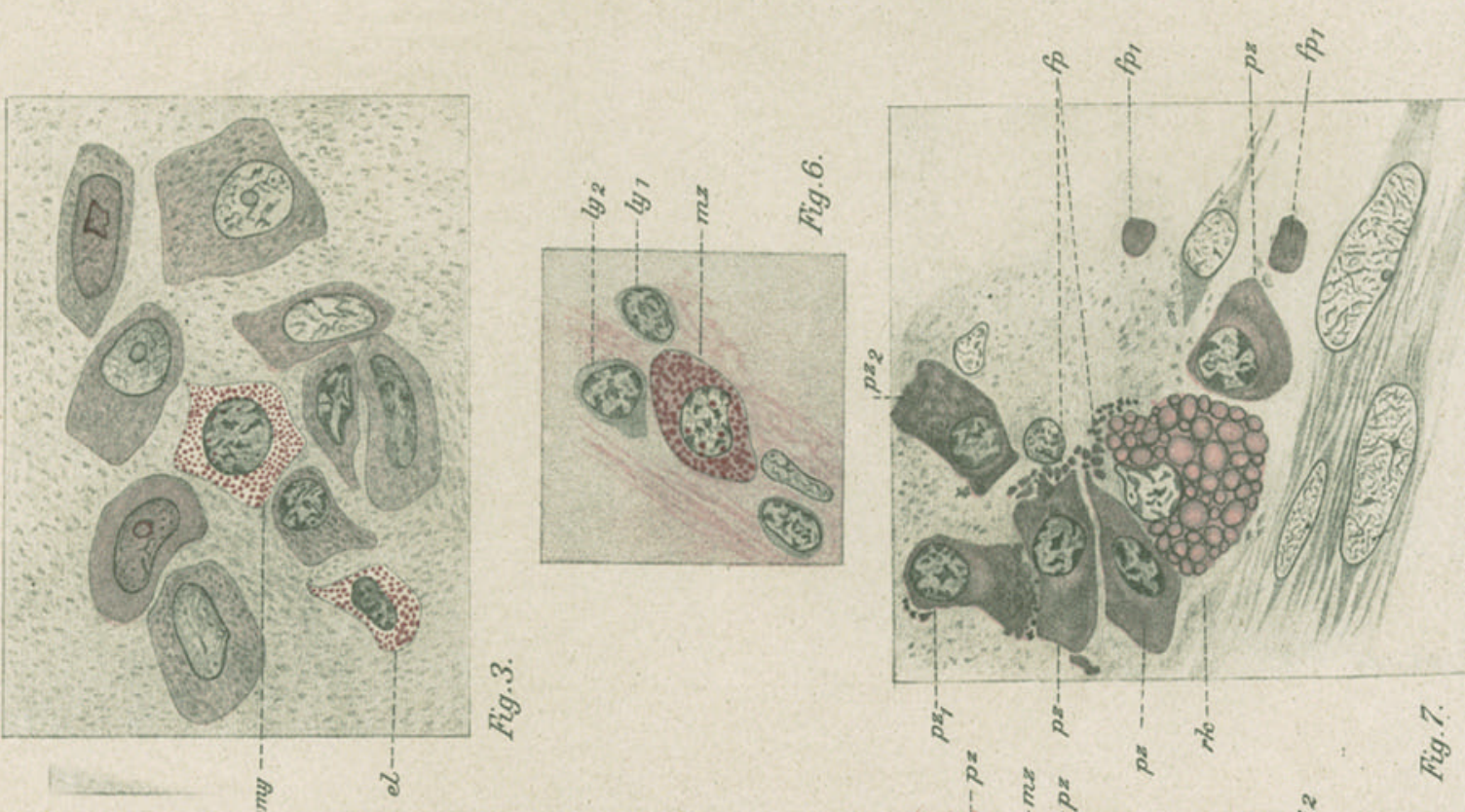

है 